\title{
Call for Papers / Appel à contributions
}

\author{
Special Issue "Situating Masculinities" \\ Guest Editors: Todd W. Reeser and Kaliane Ung \\ Deadline: March 1, 2021
}

As a bourgeoning wing of gender studies, Critical Studies on Men and Masculinities (CSMM) (or "Masculinity Studies") has been, and continues to be, deeply influenced by feminist thought. This special issue aims to place Beauvoir's corpus and her legacy into closer dialogue with CsMM, asking questions such as: In what ways and to what extent does Beauvoir theorize male or female masculinity? Do men "become" men or are they "born" men? How is Sartre's masculinity discussed? How has or could Beauvoir factor in to csMM? We welcome work in masculinity studies that does not directly treat Beauvoir. Completed papers that follow the $S d B S$ "Instructions for Authors" should be submitted on-line at www.brill.com/sdbs. Articles that are not selected for this special issue may be considered for other issues of $S d B S$.

\section{Numéro spécial «Situer les masculinités» \\ Rédacteur.trice.s invité.e.s: Todd W. Reeser et Kaliane Ung \\ Date limite: $1^{\text {er }}$ mars 2021}

Nouvelle branche des études du genre, les Critical Studies on Men and Masculinities (CSMM) ou les études de la masculinité continuent d'être profondément nourries par la pensée féministe. Ce numéro spécial souhaite faire entrer le corpus et l' héritage de Beauvoir en conversation avec les études sur la masculinité, en partant des axes de réflexion suivants : de quelle manière Beauvoir théoriset-elle la masculinité des hommes et des femmes? Les hommes sont-ils «nés» hommes ou le deviennent-ils? Qu' en est-il de la masculinité de Sartre? Quelle est l'influence de Beauvoir sur les études sur la masculinité? Nous accueillons également les travaux sur les études de la masculinité qui ne se penchent pas spécifiquement sur Beauvoir. Les articles doivent être soumis à la revue sur la plateforme numérique www.brill.com/sdbs. Les articles non sélectionnés pour ce numéro pourront être considérés pour un numéro à venir. 